\section{Wrong committees axed?}

SIR - A recent leading article (Nature 368, 482; 1994) praised the termination by the US Office of Management and Budget of a large number of scientific and technical advisory committees. On the face of it, such a move is laudable, but there are deeper implications.

For one thing, the approach taken to reducing the number of advisory committees was arbitrary and heavy-handed: the National Institutes of Health (NIH) and the Food and Drug Administration (FDA) were told to reduce their advisory committees by one-third, regardless of their merits. Many of them make a vital contribution: at FDA, they speed up and lend certainty to the regulatory review of pharmaceuticals, and at NIH they provide essential peer-review of the arcana of grant proposals and other programmes (Science 264, 191; 1994).

By contrast, panels that are clearly superfluous but 'politically correct' have been spared; NIH's Recombinant DNA Advisory Committee (RAC), which has evolved into nothing more than a de facto regulatory agency for human gene therapy proposals, is one example. On its merits, the RAC should go. First, the NIH's jurisdiction provides three additional levels of review (local institutional biosafety committees, the RAC and the NIH director) that are internally redundant and also duplicate the legally required evaluations of institutional review boards and the FDA.

Second, the RAC lacks expertise relevant to certain aspects of the regulation of pharmaceuticals, including manufacture and quality-control testing, with the nuances of clinical trials. Membership is heavy on lawyers, political scientists and ethicists.

Not surprisingly, the NIH has sometimes held the protocols to standards very different from those the FDA applies to all other experimental treatments. The result has been that researchers are confused and spend their time on paperwork instead of experiments.

Third, the NIH committee holds only a few brief meetings a year, forcing investigators to wait several months for a proposal to be considered. Moreover, because the RAC is not continuously available to them, researchers are unable to obtain permission to make important real-time adjustments to the experiment, if problems occur or if other developments dictate changes in course. The NIH committee has recently made some modest recommendations intended to streamline its regulatory review, but even if its oversight were substantially more efficient, the committee's worth would still founder on the principle that something that is not worth doing at all is not worth doing well.
Especially when the administration is applying the "reinventing government" axe to important advisory panels at the NIH and at the FDA, eliminating unnecessary layers of gene therapy regulation would be a win-win proposition: reduction of unnecessary federal and researchers' spending on regulation; more resources available for the actual research; and with diminished regulatory disincentives, greater interest in gene therapy from industry. Improvements in the trim and motion of the ship of state are important, but politicians must be circumspect about which and how much ballast to discard.

\section{Henry I. Miller}

Hoover Institution \& Institute

for International Studies,

Stanford University,

Stanford, California 94305-6010, USA

\section{ORI should stay}

SIR - In the best of times, it would be gratifying that there remain adherents to the Platonic ideals; the existence of an absolute good comes to mind. In that spirit, I wish to dissent from the opinions expressed in your leading article regarding the necessity (or lack thereof) for an Office of Scientific (now Research) Integrity (ORI) (Nature 368, 1-2; 1994).

While it is fabrication and falsification of research results that receive most attention, particularly in the popular press, plagiarism is the more egregious of common sins, particularly in research. Plagiarism is often punishable by expulsion from school or university, but, if it goes undetected, may become institutionalized and accepted (within certain confines). If plagiarism takes place in an institution of higher learning, particularly one with a relatively decentralized bureaucracy, there may be a (retaliation-free) recourse for the subject of the plagiarism. But if such an offence occurs in more 'paraacademic' institutions (museums and research institutes) wherein the hierarchical systems may be more rigid (oligarchical at times) and without the habitual academic freedoms some take for granted, the individual subject to the offence may have no such recourse.

I would therefore submit the following: your first lesson, that the ORI is not suited to the task for which it was created, is without doubt true. Your second lesson, that the ORI should be abolished, does not follow. Indeed, its mission should be expanded. At present, only institutions receiving federal funds are required to establish policies and procedures for handling misconduct allegations. Furthermore, it is the institution in which the misconduct occurred that is responsible for undertaking the investigation. And even when misconduct occurs in an institution receiving federal funds, if the particular instance of misconduct does not directly involve a definite federally funded project, then there is no recourse to the ORI. In these times of diminishing (or at least harder to obtain) federal research monies, an increasing number of potential misconduct cases are consequently free from any oversight besides the perhaps overly immoderate measure of a court of law.

\section{Luis A. Ruedas}

Thomas More College,

Ohio River Biological Station,

Route 8, Box 86, California,

Kentucky 41007-9804, USA

\section{Tumour diagnosis}

SIR - The ability to refine pathological diagnosis and improve its objectivity, particularly in the context of small biopsies and cytological material, has been dramatically demonstrated by the case of Hubert Humphrey's bladder cancer (Nature 369, $13 ; 1994)$. However, for this approach to become generally applicable in a diagnostic setting will require those tools to be cheap, easy to use, robust and applicable to hospitals and clinics without sophisticated molecular biology laboratories. PCR-based methods are complex, costly and applicable to only relatively few centres, and certainly not in district hospitals at present. Simpler methods exist that are easily and cheaply applicable to clinical samples ${ }^{1}$. The approach is based upon the remarkable correlation between overexpression of the $\mathrm{p} 53$ protein and malignancy. This has been demonstrated in a wide range of retrospective studies in a wide range of tumour types. In addition, in a large prospective study, p53 immunohistochemistry has been shown to be of real clinical utility in diagnostic cytopathology including in diagnostically difficult cases ${ }^{2}$.

Immunohistochemistry is simple, cheap and employed in nearly all pathology laboratories today. Consequently there is no reason why this simple technology could not be used as an effective adjunct to conventional morphological analysis in cytological and pathological diagnosis and screening today with the attendant positive impact on health care.

Peter A. Hall

Stephanie P. Dowell

David P. Lane

Departments of Pathology and

Biochemistry,

University of Dundee,

Dundee DD19SY, UK

1. Hall, P. A. et al. Lancet 338, 513 (1991).

2. Dowell, S. P. Cancer Res. (in the press). 